\title{
Analysis of non-isothermal fluid flow past an in-line tube bank
}

\author{
M. Alavi \& H. Goshayeshi \\ Department of Mechanical Engineering, Islamic Azad University, \\ Mashhad Branch, Iran
}

\begin{abstract}
In this paper the two-dimensional, non-isothermal fluid flow past an in-line tube bank has been numerically analyzed by the finite element method. The flow is assumed to be incompressible, laminar and unsteady. To stabilize the discretized equations of the continuity and momentum, the streamline-upwind/PetrovGalerkin scheme is employed and also the energy equation is solved using the Taylor-Galerkin method. Reynolds number of 100, Prandtl number of 0.7 , and pitch-to diameter ratios (PDRs) of 1.5 and 2.0 are chosen for this investigation. Having obtained the flow and the temperature fields, the local skin friction coefficient and the local Nusselt number are calculated for the tubes in the bundle at different times. A comparison of the present study results with the results of experiments of other investigators, showed good overall agreement between them.
\end{abstract}

Keywords: finite-element, tube bank, in-line, skin friction, Nusselt number.

\section{Introduction}

The study of the fluid flow around a tube bank, widely used in many engineering phenomena, is importance such as heat exchangers, nuclear and chemical reactors, etc. In the past it was not possible to apply numerical methods therefore, the results were drawn experimentally. It is obvious that the experimental methods are not economical and are very limited to work out. The first leaders in the experimental applications in this subject were Bergelin et al. [1] that have presented their investigation of heat transfer and fluid friction of flow across the banks of tubes. Oda et al. [2] have considered the investigation of the heat transfer processes in tube banks in cross-flow and Massey [3] has also 
predicted the flow and heat transfer in tube banks in cross-flow too. But the numerical applications for fluid flow past a tube bank could be ascribed to Launder and Massey [4], who have presented their numerical prediction of viscous flow and heat transfer in tube banks. Fujii et al. [5] have presented a numerical analysis of laminar flow and heat transfer of air in an in-line tube bank. Chen et al. [6] have carried out finite element solution of laminar, incompressible flow and heat transfer of air around three and four isothermal heated horizontal cylinders in a staggered tube bank and in an in-line tube bank, respectively. Fornberg [7] performed a numerical study of flow through one infinite row of cylinders in steady-state flow and for symmetric periodic conditions. The numerical studies were accomplished by finite difference method. Dhaubhadel et al. [8] presented a finite element solution to the problem of steady flow across an in-line bundle of cylinders for Reynolds numbers up to 400 and pitch-to-diameter ratios (PDRs) of 1.5 and 2.0. Gowda et al. [9] carried out finite element simulation of transient laminar flow past an in-line tube bank with five-tubes deep. They solved the two-dimensional unsteady Navier-Stokes and energy equations. Wang et al. [10] have done numerical analysis of forcedconvection heat transfer in laminar, two-dimensional, steady cross-flow in banks of plain tubes in staggered arrangements by finite-volume method. Recently, ElShaboury and Ormiston [11] have studied numerical analysis of forcedconvection heat transfer in laminar, two-dimensional, steady cross-flow in banks of plain tubes in square and non-square in-line arrangements by finite-volume method. In this paper we survey the numerical simulation of a two-dimensional, laminar, incompressible, and non-isothermal fluid flow and calculations are carried out for a tube bank with five-tubes deep at different times. Numerical simulation can be assumed two-dimensional flows because the length of the tube is infinite, and the fluid flow assumed to be laminar because the fundamental equations are based on laminar conditions.

\section{Governing equations and solution procedures}

The transient continuity, momentum, and energy equations in a dimensionless form for the incompressible, laminar flow of a Newtonian fluid are, respectively, given by

$$
\begin{gathered}
\nabla \cdot \overline{\mathbf{V}}=0 \\
\frac{\mathrm{D} \overline{\mathbf{V}}}{\mathrm{D} \bar{t}}=-\nabla \bar{P}+\frac{1}{\operatorname{Re}} \nabla^{2} \overline{\mathbf{V}} \\
\frac{\mathrm{D} \theta}{\mathrm{D} \bar{t}}=\frac{1}{\mathrm{Pe}} \nabla^{2} \theta
\end{gathered}
$$

where $\overline{\mathbf{V}}$ is the dimensionless velocity vector, $\bar{t}$ is the dimensionless time, $\bar{P}$ is the dimensionless pressure, Re is the Reynolds number, $\theta$ is the dimensionless temperature and $\mathrm{Pe}$ is the Peclet number. The dimensionless variables are defined as follows: 


$$
\begin{gathered}
\overline{\mathbf{V}}=\frac{\mathbf{V}}{\mathrm{U}_{\infty}}, \overline{\mathbf{X}}=\frac{\mathbf{X}}{\mathrm{D}}, \bar{t}=\frac{t \mathrm{U}_{\infty}}{\mathrm{D}}, \bar{P}=\frac{\mathrm{P}}{\rho \mathrm{U}_{\infty}^{2}}, \theta=\frac{T-\mathrm{T}_{\infty}}{\mathrm{T}_{\mathrm{w}}-\mathrm{T}_{\infty}}, \\
\operatorname{Re}_{\infty}=\frac{\rho \mathrm{U}_{\infty} \mathrm{D}}{\mu}, \operatorname{Re}_{\max }=\frac{\rho \mathrm{U}_{\max } \mathrm{D}}{\mu}, \mathrm{Pe}=\frac{\rho \mathrm{C}_{\mathrm{p}} \mathrm{U}_{\max } \mathrm{D}}{\mathrm{k}}
\end{gathered}
$$

where, $\mathbf{X}$ is the coordinate vector, $\mathrm{D}$ is the tube diameter, $t$ is the time, $\rho$ is the fluid density, $P$ is the pressure, $\mu$ is its fluid viscosity, $\mathrm{C}_{\mathrm{p}}$ is its specific heat, $\mathrm{U}_{\infty}$ is the free-stream velocity, $\mathrm{U}_{\max }$ is the average velocity at minimum flow crosssection $\left(\left(\mathrm{U}_{\max } / \mathrm{U}_{\infty}\right)=\mathrm{PDR} /(\mathrm{PDR}-1)\right), T$ is the temperature, $\mathrm{T}_{\infty}$ is the free-stream temperature, $T_{w}$ is the wall temperature and $\mathrm{k}$ is the fluid thermal conductivity.

The continuity and momentum equations are solved by Galerkin approximation and the system of discretized equations for each element is as follows:

$$
\mathbf{M}^{(\mathbf{e})} \cdot \dot{\mathbf{Z}}^{(\mathbf{e})}+\mathbf{K}^{(\mathbf{e})} \cdot \mathbf{Z}^{(\mathbf{e})}+\mathbf{F}^{(\mathbf{e})}=0
$$

where for each typical element (e), $\mathbf{M}^{(\mathbf{e})}$ is the mass matrix, $\mathbf{Z}^{(\mathbf{e})}$ is the vector of unknown variables, $\mathbf{K}^{(\mathbf{e})}$ is the stiffness matrix, containing the linear terms and $\mathrm{F}^{(\mathrm{e})}$ is the vector containing the convective nonlinear terms that is defined in reference [12]. For calculating the flow and pressure fields, the final system of equations must be solved which can achieved after assembling the system of element equations for all of this elements and therefore the Fortran program has been written. For exertion of the upwind, Petrov-Galerkin scheme is used in which the weighting function for a typical node is modified in such a way that weight of the upwind node is heavier than the downwind node [13].

To solve the energy equation and obtain the temperature field, a TaylorGalerkin scheme is used $[14,15]$. In this method, Taylor expansion with second order approximation of temperature is used according to time. The discretized equation for a typical element is as follows:

$$
\left(\frac{1}{\Delta \mathrm{t}} \mathbf{M}^{(\mathrm{e})}+\frac{1}{2 \mathrm{Pe}} \mathbf{K}_{\mathrm{d}}^{(\mathrm{e})}\right) \theta^{(\mathrm{e})^{\mathrm{n}+1}}=\left[\frac{1}{\Delta \mathrm{t}} \mathbf{M}^{(\mathrm{e})}-\frac{1}{2 \mathrm{Pe}} \mathbf{K}_{\mathrm{d}}^{(\mathrm{e})}-\left(\mathbf{K}_{\mathrm{a}}^{(\mathrm{e})}+\mathbf{K}_{\mathrm{bd}}^{(\mathrm{e})}\right)\right] \theta^{(\mathrm{e})^{\mathrm{n}}}
$$

where, $\mathrm{M}^{(\mathrm{e})}, \mathrm{K}_{\mathrm{d}}^{(\mathrm{e})}, \mathrm{K}_{\mathrm{a}}^{(\mathrm{e})}, \mathrm{K}_{\mathrm{bd}}^{(\mathrm{e})}$ are the mass, the diffusion, the advection and the balancing diffusion matrices for element, respectively. In all the above calculations, continuity, momentum and energy equations are solved in the unsteady form with dimensionless time step of 0.01. Assuming that the transport properties are constant in each time step, continuity and momentum equations are solved first and then by using the obtained flow field, energy equation is solved and the temperature field is computed. These calculations are repeated in the successive time steps until the steady-state are obtained.

\section{Physical model and boundary conditions}

Physical model of flow around an in-line tube bank with five tubes in the flow direction and with depth of one is shown in figure 1. In this model, according to reference [16], the flow field is considered 5 times bigger than the tube diameter in the up-stream $\left(\mathrm{L}_{\mathrm{us}}\right)$ and 20 times bigger than the tube diameter in down-stream $\left(\mathrm{L}_{\mathrm{ds}}\right)$. 


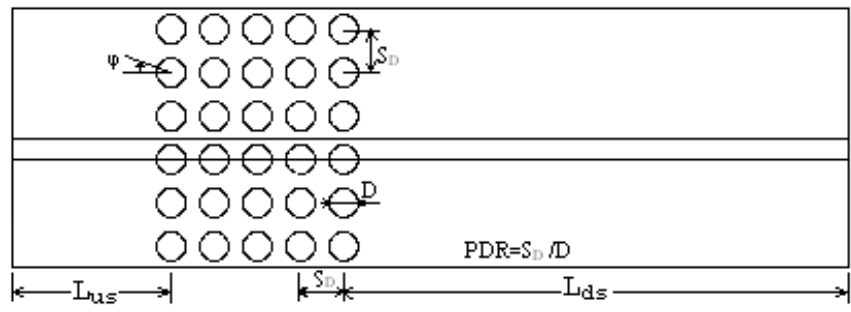

Figure 1: $\quad$ Physical model of flow around an in-line tube bank.

The computational domain and the tube bank boundary condition used in this work are also shown in figure 2. This domain is divided into elements which are isoperimetric rectangular with four nodes. The velocity and the temperature in four nodes of each element are calculated, as well as its centre of pressure. Some of the elements of the mesh around the tubes are shown in figure 3. At first, three grids of coarse with the number of 6869 , medium with the number of 12684 and fine with the number of 21679 of elements is used in the computations until mesh optimization was made and higher than these elements the results are the same. As it can be seen, around the tubes where the velocity and the temperature gradient are greater, the finer mesh is adapted.

The no-slip and the constant temperature, $\mathrm{T}=\mathrm{T}_{\mathrm{w}}$, boundary conditions are applied on the surface of the tube. At the inflow, the $x$-component of the velocity vector and the temperature are set equal to the free-stream velocity, $\mathrm{U}_{\infty}$, and the free-stream temperature, $\mathrm{T}_{\infty}$, respectively. At the outlet, the velocity gradient $u$ and the temperature gradient in direction $x$ and $v$-component of velocity equals zero, i.e. fully-developed boundary conditions are enforced. In the axis of symmetry, the velocity gradient $u$ and the temperature gradient in direction $y$ and so $v$-component of velocity equals zero. Moreover, as a reference value, the pressure is set equal to the zero at the outflow. The initial conditions are

$$
\begin{aligned}
& \overline{\mathrm{V}}(\mathbf{X}, 0)=0 \\
& \theta(\mathbf{X}, 0)=0
\end{aligned}
$$

The mathematical model of the problem is expressed by the coupled system of eqns (1) through (3) and the initial conditions (7) and (8) together with the boundary conditions are shown in figure 2 . Reynolds number of 100 , Prandtl number of 0.7 , and PDRs of 1.5 and 2.0 are chosen for the investigation.

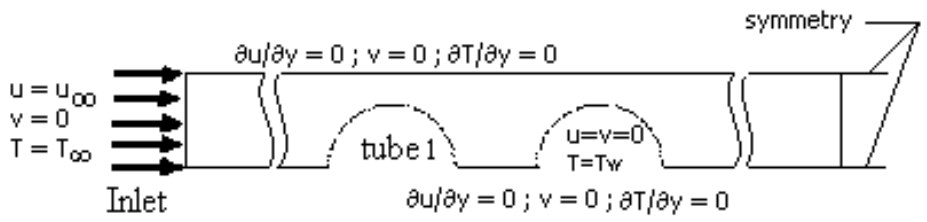

Figure 2: Boundary conditions in the computational domain. 


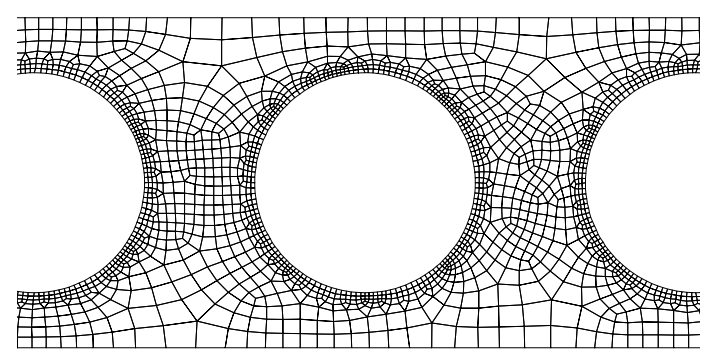

Figure 3: A portion of finite element mesh in in-line array.

\section{Results and discussion}

\subsection{Streamlines}

In figure 4 the streamlines for flow past an in-line tube bank with five tubes in the flow direction with Reynolds numbers 100 , and $\mathrm{PDR}=1.5$ at different times until the steady-state reaches, are shown. At the beginning when the flow comes into contact with the tubes, its presence will not be felt by the tubes. Hence, the inertia forces dominate the viscous forces and the streamline pattern resembles that of an invicid flow (figure 4a). At $\mathrm{t}=0.5$ the symmetry is lost and the incipience of separation can be seen on all the tubes (figure 4b). A pair of symmetric eddies normally forms behind the tubes. The eddy in which the fluid circulates keeps growing with time until it reaches the steady-state (figure 4c).

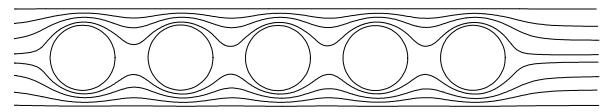

$$
\overline{\bar{t}}=0.1
$$

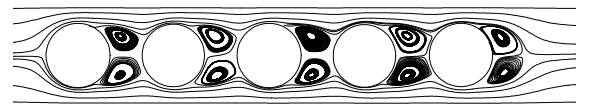

$$
\bar{t}=0.5
$$

(b)

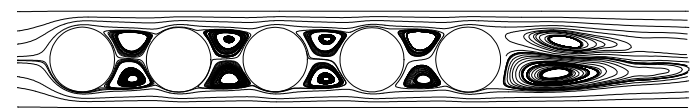

Steady-state

(c)

Figure 4: $\quad$ Streamlines in the in-line tube bank for PDR $=1.5$.

\subsection{Isotherms}

The isotherms for flow past an in-line tube bank with five tubes in the flow direction with Reynolds numbers 100 , and $P D R=1.5$ at different times until the steady-state, are shown in figure 5 . It is possible to predict the amount of heat 
flow at different times and in the various points of the tube bank. It is obvious that in places where isotherms are closer to each other, the temperature gradient is greater and heat transfer is higher. The isotherms at $\bar{t}=0.1$ of figure 5(a) are crowded over the entire tubes and are symmetrical. As time passes, this symmetry is lost because of the recirculation region between the tubes (figure 5(b)). The growth of isotherms follows the growth of streamlines. In figure 5(c), it can be seen that, in the steady-state, the isotherms are crowded over the front half of the first tube that indicate a high radial heat flux and the temperature difference is around 300 to $400 \mathrm{~K}$. But over the other tubes, low velocity recirculation flow interacts with parts of the front half of the subsequent tubes indicate lower radial heat flux.

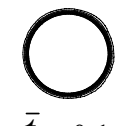

$\bar{t}=0.1$

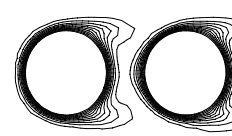

$\bar{t}=0.5$
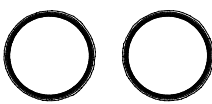

(a)
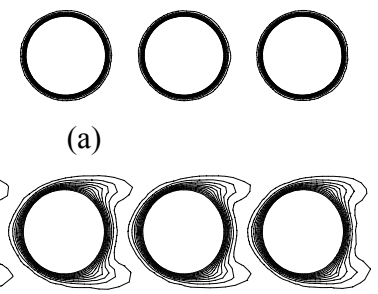

(b)

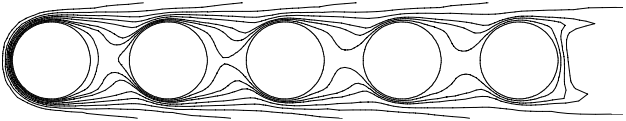

Steady-state

(c)

Figure 5: Isotherms in the in-line tube bank for PDR $=1.5$.

\subsection{Skin friction coefficient}

Shearing action between the fluid and the tube surface, which is also known as local skin friction coefficient has been investigated. For laminar and incompressible flow past a tube bank, after calculations of the flow field for each tube, the local skin friction coefficient $\left(C_{\mathrm{f}}\right)$ is defined as:

$$
C_{\mathrm{f}}=\frac{\tau_{\mathrm{w}}}{\frac{1}{2} \rho\left(\mathrm{V}_{\text {max }}\right)^{2}}=\left.\frac{2}{\operatorname{Re}_{\text {max }}} \frac{\partial \overline{\mathrm{V}}_{\mathrm{t}}}{\partial \mathrm{n}}\right|_{\mathrm{w}}
$$

where, $\tau_{\mathrm{w}}$ is the tube wall shear stress, $\overline{\mathrm{V}}_{\mathrm{t}}$ is the dimensionless tangent velocity vector and $\overline{\mathrm{n}}$ is the dimensionless normal vector on surface.

Figure 6 shows these coefficients for PDR of 1.5 at the steady-state. At the first instance, the distribution of friction coefficient is the same as that for all the tubes. This is because the flow initially behaves like a potential flow. As time passes, the local skin friction coefficient decreases and for the first tube is different from those for the following tubes. The maximum local skin friction coefficient is the same for all the tubes, except the first tube that it is a little 
bigger and it is almost for an angle of $80^{\circ}$. For the tubes, except the first tube, the minimum value of $C_{\mathrm{f}}$ that is initially on the rear half of the cylinder has shifted to the front portion of the tube with progress in time, and it is for an angle of $30^{\circ}$. Figure 7 shows local skin friction coefficients for PDR of 2.0 at the steady-state. As it can be seen, those decrease over all the tubes compare to PDR of 1.5, because the velocity gradients are lower at higher PDRs. In this figure, the $C_{f}$ results are analoged with Ref. [12] and show good overall agreement.

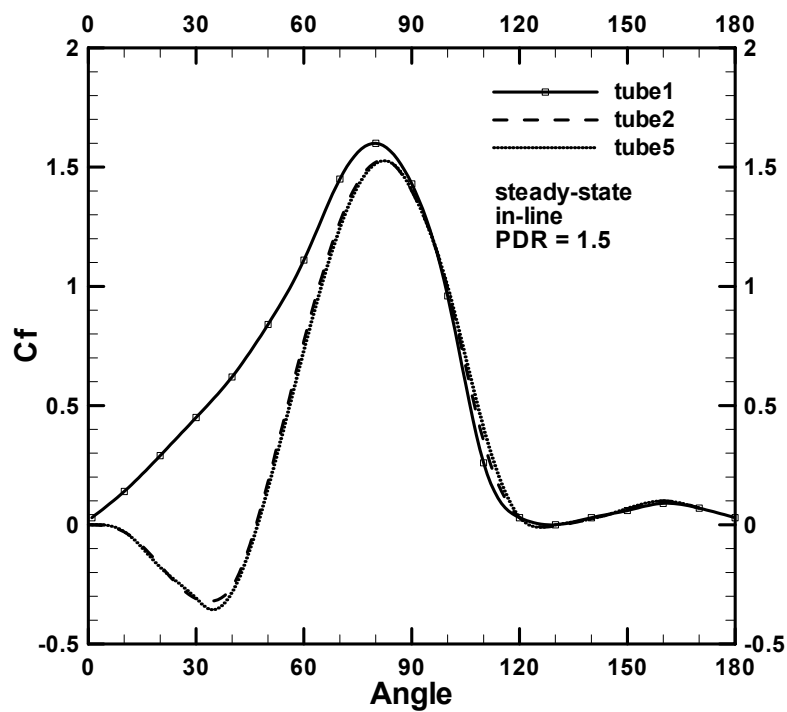

Figure 6: The local skin friction coefficients for $\mathrm{PDR}=1.5$.

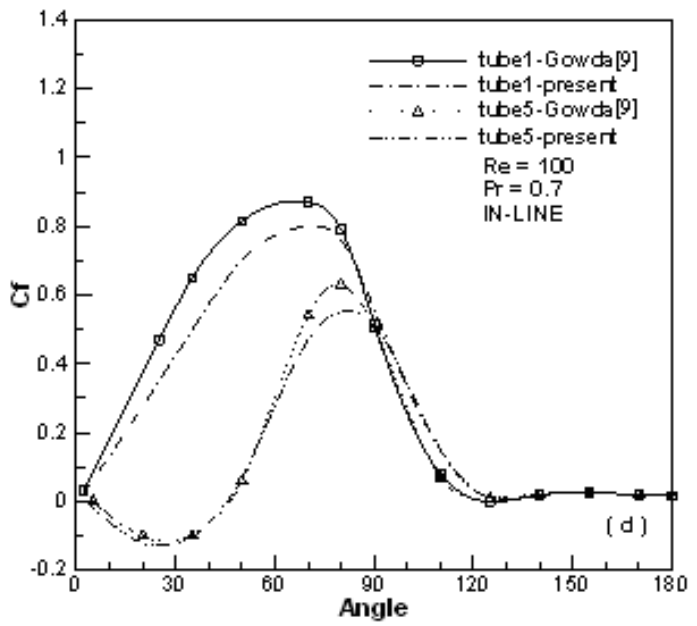

Figure 7: The local skin friction coefficients for PDR $=2.0$ at the steadystate [9]. 


\subsection{Nusselt number}

Nusselt number is one of the vital parameters of interest to the designer. The local Nusselt number and the bulk temperature are defined as:

$$
\begin{gathered}
\mathrm{Nu}=-\frac{\mathrm{D}}{\left(\mathrm{T}_{\mathrm{w}}-\mathrm{T}_{\mathrm{b}}\right)}\left(\left.\frac{\partial T}{\partial \mathrm{n}}\right|_{\mathrm{w}}\right)=-\frac{1}{1-\theta_{\mathrm{b}}}\left(\left.\frac{\partial \theta}{\partial \overline{\mathrm{n}}}\right|_{\mathrm{w}}\right) \\
\mathrm{T}_{\mathrm{b}}=\frac{\int_{\mathrm{D} / 2}^{\mathrm{S}_{\mathrm{D}} / 2} \rho u T \mathrm{dy}}{\rho\left(\mathrm{S}_{\mathrm{D}} / 2\right) \mathrm{U}_{\infty}}
\end{gathered}
$$

where $\mathrm{D}$ is the tube diameter, $\mathrm{T}_{\mathrm{w}}$ is the wall temperature, $\mathrm{T}_{\mathrm{b}}$ is the bulk temperature at minimum cross-section and $\mathrm{S}_{\mathrm{D}}$ is the transverse pitch.

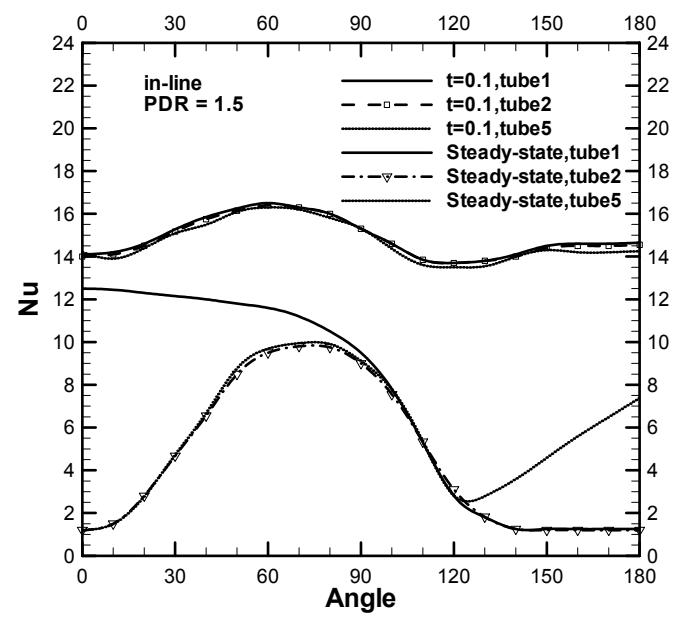

Figure 8: The local Nusselt number for PDR $=1.5$ at the time $=0.1$ and the steady-state.

Figure 8 shows the distribution of the local Nusselt number around the tubes for PDR of 1.5 at dimensionless time 0.1 , and for the steady-state. In the initial phase, $\bar{t}=0.1$, the distribution of local Nusselt number is almost the same on all the tubes; on the other hand, the temperature gradient is intense around the tubes, thus the Nusselt number is great. As time passes, the local Nusselt number decreases and for the first tube differs from the second and subsequent tubes. As a result, the thinner boundary layer over the first tube leads to a higher temperature gradient and, thus, to a higher heat transfer rate at the tube surface. The maximum Nusselt number for the first tube occurs in the region of the front stagnation point, and then as the fluid moves, it decreases with the growth of thermal boundary layer and for the next tubes occurs at around angle of $60^{\circ}$. The minimum Nusselt number for the first tube corresponds to the boundary layer separation point; but for the next tubes Nusselt number is minimum in the stagnation point because it occurs in the recirculation region behind tube. 
Figure 9 shows the distribution of the local Nusselt number around the tubes 1, 2 and 5 at the steady-state for PDR $=2.0$. As it can be seen, those decrease over all the tubes. In this figure 13b, $N u$ results are compared with [12,13] and are shown good overall agreement.

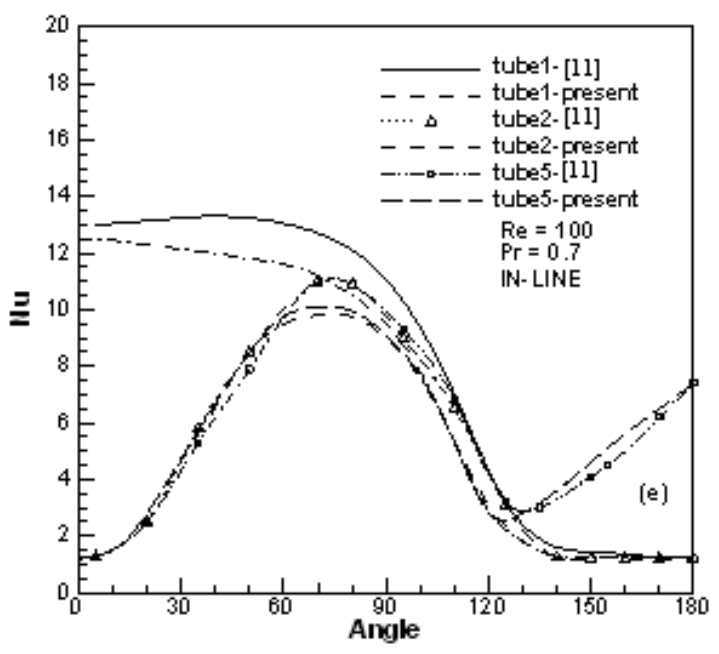

Figure 9: $\quad$ The local Nusselt number for PDR $=2.0$ at the steady-state [11].

\section{Conclusions}

The problem of the two-dimensional non-isothermal fluid flow past an in-line tube bank is numerically simulated by the finite element method. The flow was assumed to be incompressible, laminar and unsteady. Reynolds number of 100, Prandtl number of 0.7 , and PDRs of 1.5 and 2.0 are chosen for the investigation.

At the beginning when the flow comes into contact with the tubes, its presence will not be felt by the tubes. The distribution of friction coefficient and the distribution of local Nusselt number is almost the same on all the tubes. Hence, the inertia forces dominate the viscous forces and the streamline pattern resembles that of an invicid flow. At the first instance, the isotherms are crowded over the entire tubes and are symmetrical and the temperature gradient is intense around the tubes, thus the Nusselt number is great. As time passes, a pair of symmetric eddies normally forms behind the tubes and they grow with time until the flow reaches the steady-state and also the local skin friction coefficient and the local Nusselt number decrease until they reach steady-state. For the tubes, except the first tube, the minimum value of the local skin friction coefficients that is initially on the rear half of the cylinder has shifted to the front portion of the tube with progress in time. The growth of isotherms follows the growth of streamlines. At the steady-state, the thinner boundary layer over the first tube leads to a higher temperature gradient and, thus, to a higher heat transfer rate at the tube surface, the maximum Nusselt number for the first tube occurs in the 
region of the front stagnation point, and then as the fluid moves, it decreases with the growth of thermal boundary layer and for the next tubes occurs at around angle of $60^{\circ}$, the minimum Nusselt number for the first tube corresponds to the boundary layer separation point; but for the next tubes Nusselt number is minimum in the stagnation point because it occurs in the recirculation region behind tube.

The local skin friction coefficients and the local Nusselt number are found to be dependent on pitch to diameter ratios (PDRs) and are higher for smaller PDRs and vice versa. A comparison of the present study results with the results of experiments of other investigators, showed good overall agreement between them.

\section{References}

[1] Bergelin, O.P., Brown, G.A. \& Doberstein, S.C., Heat transfer and fluid friction during flow across banks of tubes - IV. Trans. ASME, 74, pp. 953960, 1952.

[2] Oda, S., Kostic, Z.G. \& Sikmonovic, S., Investigation of the heat transferprocesses in tube banks in cross-flow. Int. Seminar on Recent Developments in Heat Exchangers, Trogir, Yugoslavia, 1972.

[3] Massey, T.H., The prediction of flow and heat fransfer in banks of tubes in cross-flow. Ph.D. Thesis, Central Electricity Research Laboratories, Leatherhead, Surrey, February, 1976.

[4] Launder, B.E. \& Massey, T.H., The numerical prediction of viscous flow and heat transfer in tube banks. Trans. ASME., J. Heat Transfer, 100, pp. 565-571, 1978.

[5] Fujii, M., Fujii, T. \& Nagata, T., A numerical analysis of laminar flow and heat transfer of air in an in-line tube bank. Numer. Heat Transfer, 7, pp. 89109, 1984.

[6] Chen, C.K., Wong, K.L. \& Cleaver, J.W., Finite element solutions of laminar flow and heat transfer of air in a staggered and an in-line tube bank. Int. J. Heat and Fluid Flow, 7, pp. 291-300, 1986.

[7] Fornberg, B., Steady incompressible flow past a row of circular cylinders. J. Fluid Mech, 225, pp. 655-671, 1991.

[8] Dhaubhadel, M.N., Reddy, J.N. \& Telionis, D.P, Penalty finite element analysis of coupled fluid flow and heat transfer for in-line bundle of cylinders in cross-flow. J. Nonlinear Mech, 21, pp. 361-373, 1986.

[9] Gowda, Y.T.K., Patnaik, B.S.V.P., Narayana, P.A.A. \& Seetharamu, K.N., Finite element simulation of transient laminar flow and heat transfer past an in-line tube bank. Int. J. Heat and Fluid Flow, 19, pp. 49-55, 1998.

[10] Wang, Y.Q., Penner, L.A. \& Ormiston, S.J., Analysis of laminar forced convection of air dor cross-flow in banks of staggered tubes. Numer. Heat transfer, Part A, 38, pp. 819-845, 2000.

[11] El-Shaboury, A.M.F. \& Ormiston, S.j., Analysis of laminar forced convection of air cross-flow in in-line tube banks with nonsquare arrangements. Numer. Heat Transfer. Part A, 48, pp. 99-126, 2005. 
[12] Heinrish, J.C. \& Pepper, D.W., Intermediate finite element method, fluid flow and heat transfer applications. CHP 10, pp. 209-255, 1999.

[13] Brooks, A.N. \& Hughes, T.J.R., Streamline-upwind/Petrov-Galerkin formulations for convection dominated flows with particular emphasis on the incompressible Navier-Stokes equations. Computer Methods in Applied Mech. and Eng, 32, pp. 199-259, 1982.

[14] Usmani, A.S., Cross, J.T. \& Lewis, R.W., A finite element model for the simulations of mould filling in metal casting and the associated heat transfer. Int. J. Numer. Meth. in Eng, 35, pp. 788-806, 1992.

[15] Arefmanesh, A. \& Alavi, M.A., A hybrid finite difference-finite element method for solving the 3-D energy equation in non-isothermal flow past over a tube. Int. J. of Numer. Meth. for Heat and Fluid Flow, 18, pp. 50-66, 2008.

[16] Tezduyar, T.E. \& Shih, R., Numerical experiments on downstream boundary of flow past cylinders. J. Engn. Mech, 117, pp. 854-871, 1991. 